\title{
Study on Dynamic Evaluation Method of Stability of Slope Controlled by Structural Plane Based on Deformation Characteristics
}

\author{
Qun $\mathrm{Luo}^{1}$, Tianlong Zhao ${ }^{2,3, *}$ \\ ${ }^{1}$ China Railway Fourth Survey and Design Institute Group Co., Ltd., Wuhan, Hubei, China \\ ${ }^{2}$ College of River and Ocean Engineering, Chongqing Jiaotong University, Chongqing, China \\ ${ }^{3}$ Diagnostic Technology on Health of Hydraulic Structures Engineering Research Center of Chongqing Education Commission of \\ China, Chongqing Jiaotong University, Chongqing, China
}

\begin{abstract}
The study on deformation characteristics of structural plane is the research foundation of structural plane mechanics and hydraulics. In this paper, a calculation method for dynamic evaluation of the stability of slope controlled by structural plane based on deformation characteristics was proposed on the basis of shear reinforcement three-fold line constitutive model, and it was applied to the example of Raytheon landslide in Chongqing-Guizhou high-speed railway. By comparison, the calculated results were in good agreement with the measured displacement results, and the displacement development process of the dangerous rock mass can be well simulated, thus verifying the feasibility and engineering practicality of the method.
\end{abstract}

\section{Introduction}

Structural plane refers to the geological interface or zone formed in a rock mass with a certain direction of extension and a relatively small length and thickness during the historical development of natural geological body, such as bedding plane, unconformity plane, joint plane, fault and schistosity plane. The texture of rock mass is very complex, and the rock mass structure is composed of various structural planes and rocks with different sizes and origins. The deformation, failure mechanism and mechanical criterion of rock mass are controlled by the structural plane in rock mass and the rock mass structure formed by its cutting (Liu et al., 2012; Wang\&Jiang,2018). As early as the 1950s, the Austrian School represented by Muller (Chen et al.,2013) recognized the mechanical properties of rock mass in structural plane and its control over engineering stability. In the 1970s, Gu (1979) made an in-depth analysis of the rock mass structure, and further clearly put forward the basic concepts of engineering geological rock group, structural plane and structural body, and made a comprehensive and in-depth exposition of their causes, classification and classification.

The existence of structural surfaces greatly affects the mechanical properties and stability of rock mass, and the stability and damage patterns of rock slope are largely determined by the controlling structural plane in the rock mass. Therefore, the mechanical properties of structural plane have very important value of engineering practice in the study of rock slope stability, and scholars in various countries have also carried out a lot of research work in this regard, and nowadays many important research results have been achieved (Wang et al.,2020). In view of the plane sliding problem and sliding problem of wedge body, Qi et al. (2007) proposed an algorithm for seismic permanent displacement of rocky slopes considering structural plane degradation on the basis of previous studies. Zhao et al (2008) established a structural rock slope stability evaluation model based on the paradigm inference method with model similarity priority. In allusion to the shortcomings of establishing the constitutive model of rock mass structural plane within the theoretical framework of classical continuum medium mechanics, Xu and Ren (2011) established a new rock mass structural plane constitutive model by direct method based on the actual stress and deformation characteristics of rock mass structural plane. Wan et al. (2019) proposed that the polar projection stereographic projection method can be used to evaluate and analyze the stability of high and steep rock slope with multiple structural planes in alpine valley area.

At present, the research on the mechanical properties of the structural plane focuses on the deformation and strength of the structural plane, and the study of the deformation characteristics of the structural plane is the basis of the mechanical and hydraulic characteristics of the structural plane. In this paper, a shear-strengthened trilinear shear constitutive model is proposed, and the sliding displacement process of the upper rock mass of the slope controlled by the structural plane caused by the deformation characteristics of the model under excavation unloading is studied. Then, the excavation displacement model of slope controlled by structural plane is established to analyze the dynamic stability of slope, and select a slope in southwest China as an example to verify the feasibility

\footnotetext{
*Corresponding author: neo_3303@163.com
} 
and engineering applicability of the calculation model and stability analysis method.

\section{Slope motion equation by excavation unloading}

The rock structural plane has a certain degree of fluctuation and roughness. For the weak structural plane with interlayer that contains a certain amount of gravel, and the material nonhomogeneity of the structural plane is strong. In the previous study on the shear strength characteristics of the structural plane, the authors found that during the shear dislocation process of the upper and lower rock masses, there was a process of particle rearrangement in the initial stage of shear to adjust the stress and then play a large shear strength. At this stage, the shear stiffness of structural plane is small, and then the shear stiffness increases significantly until the peak shear resistance. The shearing process can be generalized to a three-fold line model, as shown in figure 1.

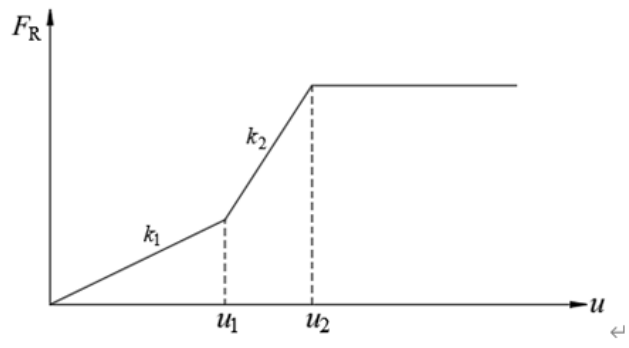

Fig. 1. Schematic diagram of shear-strengthened three-fold line constitutive model.

The shear model is divided into two stages of linear deformation. The first stage is the process of stress adjustment of structural plane to play a larger shear resistance under the action of shear force; the second section is the shear strengthening stage, in which the shear resistance increases rapidly. After reaching the peak, the shear resistance remains steady with the increase of displacement.

The relationship between sliding resistance and displacement shown in figure 1, which can be expressed by Eq. (1):

$$
F_{R}=\left\{\begin{array}{l}
k_{1} L u, u \in\left[0, u_{1}\right) \\
L\left(k_{2} u-k_{2} u_{1}+k_{1} u_{1}\right), u \in\left[u_{1}, u_{2}\right) \\
L\left(k_{2} u_{2}-k_{2} u_{1}+k_{1} u_{1}\right), u \in\left[u_{2},+\infty\right)
\end{array}\right.
$$

where $F_{R}$ is the shear resistance that can be provided by the structural plane, $k_{1}$ is the material shear modulus of the stress adjustment stage; the shear displacement of this stage is $u_{1} ; k_{2}$ is the shear modulus of the shear resistance growth stage; the shear displacement of this stage is $u_{2}$; and $L$ is the length of the structural plane. Eq. (1) is the shear-strengthened three-fold line constitutive model. This paper mainly studies the slope displacement model based on shear-strengthened three-fold line constitutive model under excavation unloading conditions.

\subsection{Calculation model for sliding resistance}

The slope with unit length along the slope direction is selected to study, and the problem is simplified to a twodimensional form. Assuming that there are unloading cracks in the back edge of the slope top, the cracks run through the structural plane, the direction is vertical, and the length is $h$. Then, taking the intersection of structural plane and fracture as the origin, the coordinate system is established along the structural plane and its normal direction, and the geological model of slope controlled by structural plane is generalized as shown in figure. 2 .

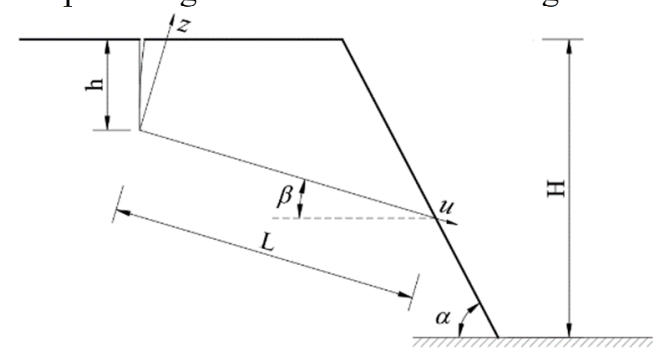

Fig. 2. Schematic diagram of slope displacement calculation.

Where $\alpha$ is the slope angle, $H$ is the slope height, $\beta$ is the dip angle of the structural plane, $L$ is the calculated length of the structural plane.

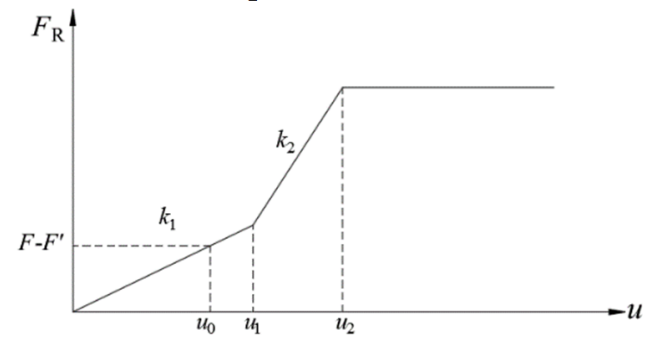

Fig. 3. Schematic diagram of shear resistance calculation.

The shear-strengthened three-fold line constitutive model is shown in Eq. (1). Under the working condition of excavation unloading, assuming the unloading load at the leading edge of potential sliding body is $F^{\prime}$, the sliding resistance decreases $F^{\prime}$, At this time, the sliding resistance calculation model is shown as the virtual line in figure 3 .

The self-weight of sliding body is expressed by Eq. (2):

$$
W=\gamma\left(\begin{array}{l}
h L \cos \beta+\frac{L^{2}}{2} \sin \beta \cos \beta \\
-\frac{h^{2}}{2} \cot \alpha-h L \sin \beta \cot \alpha \\
-\frac{L^{2}}{2} \sin ^{2} \beta \cot \alpha
\end{array}\right)
$$

The slope is in a static state before excavation, and the sliding resistance and the sliding force of the structural plane are equal to $F$. When the sliding body displacement occurs, the structural plane still exerts shear resistance, which is between 0 and sliding force, not 0 .Therefore, when the slope angle is excavated, and the load $F^{\prime}$ is removed, the stress state of the sliding body is at $\left(u_{0}, F-F^{\prime}\right)$ point in figure 3 . where $u_{0}$ and $F$ are expressed by Eq. (3): 


$$
\left\{\begin{array}{l}
u_{0}=\frac{W \sin \beta}{k_{1} L} \\
F=W \sin \beta
\end{array}\right.
$$

Unloading action will be produced after slope toe excavation. Unbalanced force $\left(F-F^{\prime}\right)$ generated by unloading, the driving force of sliding body instability, which in the curve corresponds to a displacement $u_{0}$, the value is the initial 0 point of the slide displacement. Therefore, the displacement process of the upper sliding body of the slope controlled by the structural plane caused by excavation unloading starts from $u_{0}$, and the shearresistant constitutive model becomes:

$$
F_{R}{ }^{\prime}=\left\{\begin{array}{l}
k_{1} L\left(u+u_{0}\right), u \in\left[0, u_{1}-u_{0}\right) \\
L\left[k_{2}\left(u+u_{0}\right)-k_{2} u_{1}+k_{1} u_{1}\right], u \in\left[u_{1}-u_{0}, u_{2}-u_{0}\right) \\
L\left(k_{2} u_{2}-k_{2} u_{1}+k_{1} u_{1}\right), u \in\left[u_{2}-u_{0},+\infty\right)
\end{array}\right.
$$

\subsection{Motion equation of slope controlled by structural plane}

For the calculation of displacement process of slope sliding body controlled by structural plane caused by unloading, the following assumptions exist.

(a) The sliding block analyzed is a rigid body, without considering the deformation in the displacement process.

(b) Ignoring the effect of stresses on both sides of the sliding body on its displacement process and stability.

(c) The sliding displacement of the block during unloading is not considered.

(d) Ignoring the time consuming of unloading process, the starting point of time calculation begins at the end of unloading.

Assuming that the sliding force satisfies $F \leq F_{R}\left(u_{1}\right)$, as shown in figure 3 , the displacement process model of the sliding body needs to be calculated in three stages.

\subsubsection{For $u \in\left[0, u_{1}-u_{0}\right)$}

In the time infinitesimal $d t$, the slider motion satisfies Newton's second law.

$$
F=\sum F_{i}=m a=m \frac{d^{2} u}{d t^{2}}
$$

transforming Eq. (5) to Eq. (6).

$$
d^{2} u=\frac{F-F_{R}}{m} d t^{2}
$$

Replacing Eq. (3), (4) with (6) and getting Eq. (7).

$$
d^{2} u=\frac{W \sin \beta+F^{\prime}-k_{1} L u_{0}-k_{1} L u}{m} d t^{2}
$$

Supposing the $I, J$ are as follows:

$$
\left\{\begin{array}{l}
I=\frac{k_{1} L}{m} \\
J=\frac{W \sin \beta+F^{\prime}-k_{1} L u_{0}}{m}
\end{array}\right.
$$

Then Eq. (7) can be organized as Eq. (9).

$$
\frac{d^{2} u}{d t^{2}}+I u=J
$$

Because the displacement and velocity are 0 when the sliding body begins to slide, the displacement process of the slope controlled by the structural plane caused by excavation can be described by Eq. (10).

$$
\left\{\begin{array}{l}
\frac{d^{2} u}{d t^{2}}+I u=J, u \in\left[0, u_{1}-u_{0}\right) \\
\frac{d u}{d t}=0, t=0 \\
u=0, t=0
\end{array}\right.
$$

The displacement process of the upper sliding body of the slope controlled by the structural plane caused by excavation condition can be transformed into the initial value problem of ordinary differential equation.

The control equation of Eq. (10) is a second-order linear nonhomogeneous differential equations with constant coefficients, and its general solution is:

$$
u=\bar{u}+u^{*}
$$

Where $\bar{u}$ is general solution of the homogeneous Eq. (12) corresponding to the control equation. $u^{*}$ is the special solution of the original non-homogeneous equation.

$$
\frac{d^{2} u}{d t^{2}}+I u=0
$$
(12).

Firstly, the characteristic equation is obtained by Eq.

$$
r^{2}+I=0
$$

A pair of conjugate complex roots can be obtained by solving the above characteristic equation.

$$
r_{1,2}= \pm i \sqrt{I}
$$

Therefore, the general solution of Eq. (12) can be written in the following form:

$$
\bar{u}=C_{1} \cos (\sqrt{I} t)+C_{2} \sin (\sqrt{I} t)
$$

where $C_{1}$ and $C_{2}$ are the integration constants. The following is to solve the special solution $u^{*}$, assuming that the form of non-homogeneous term is:

$$
f(t)=e^{\lambda t} P_{m}(t)
$$

Whereupon,

$$
\left\{\begin{array}{l}
\lambda=0 \\
m=0
\end{array}\right.
$$

Since it is not the root of the characteristic Eq. (13), the characteristic solution of the original non-homogeneous equation can be written as:

$$
u^{*}=C_{3}
$$

It is easy to get Eq. (10) by substituting it into Eq. (10).

$$
C_{3}=\frac{J}{I}
$$

Therefore, by substituting Eq. (15) and Eq. (19) into Eq. (11), the general solution of the non-homogeneous equation can be written as:

$$
u=C_{1} \cos (\sqrt{I} t)+C_{2} \sin (\sqrt{I} t)+\frac{J}{I}
$$

From the initial condition $u(0)=0,\left.\quad \frac{d u}{d t}\right|_{t=0}=0$, the general solution of Eq. (10) can be obtained as: 


$$
u=\frac{J}{I}[1-\cos (\sqrt{I} t)]
$$

Where, $I, J$ can be calculated by Eq. (8). The above formula is the sliding body displacement equation under the condition of $u \in\left[0, u_{1}-u_{0}\right)$.

\subsubsection{For $u \in\left[u_{1}-u_{0}, u_{2}-u_{0}\right)$}

Firstly, the end-point duration and velocity of the previous stage are calculated according to Eq. (21), which is used as the initial condition for establishing the displacement equation of the sliding body at this stage. Substitute Eq. (21) to get Eq. (22).

$$
t_{1}=I^{\frac{-1}{2}} \arccos \left[1-\frac{I\left(u_{1}-u_{0}\right)}{J}\right]
$$

From Eq. (21), the sliding velocity of sliding body at any time can be obtained as:

$$
v=\frac{d u}{d t}=\frac{J}{\sqrt{I}} \sin (\sqrt{I} t)
$$

At $t=t_{1}$, there are:

$$
v\left(t_{1}\right)=\left.\frac{d u}{d t}\right|_{t=t_{1}}=\frac{J}{\sqrt{I}} \sin \left(\sqrt{I} t_{1}\right)
$$

where $t_{1}$ is obtained from Eq. (22). Again, Eq. (3) and Eq. (4) are substituted into Eq. (6) and calculated to obtain Eq. (23).

$$
d^{2} u=\frac{W^{\prime} \sin \beta+F^{\prime}-k_{2} L u_{0}+k_{2} L u_{1}-k_{1} L u_{1}-k_{2} L u}{m} d t^{2}
$$

Supposing the $M, N$ are as follows:

$$
\left\{\begin{array}{l}
M=\frac{k_{2} L}{m} \\
N=\frac{W^{\prime} \sin \beta+F^{\prime}-k_{2} L u_{0}+k_{2} L u_{1}-k_{1} L u_{1}}{m}
\end{array}\right.
$$

So Eq. (25) can be organized as Eq. (27)

$$
\frac{d^{2} u}{d t^{2}}+M u=N
$$

When the sliding body starts at this stage, i.e. $t=t_{1}$, the displacement and velocity are respectively:

$$
\left\{\begin{array}{l}
u\left(t_{1}\right)=u_{1}-u_{0} \\
v\left(t_{1}\right)=\frac{J}{\sqrt{I}} \sin \left(\sqrt{I} t_{1}\right)
\end{array}\right.
$$

Let $T=t-t_{1}$ and then $t=T+t_{1}$ be substituted into the initial condition of Eq. (27) in parallel. The displacement process of slope sliding controlled by structural plane caused by excavation can be described by the following equation.

$$
\left\{\begin{array}{l}
\frac{d^{2} u}{d T^{2}}+M u=N, u \in\left[u_{1}-u_{0}, u_{2}-u_{0}\right) \\
\frac{d u}{d T}=\frac{J}{\sqrt{I}} \sin \left(\sqrt{I} t_{1}\right), \mathrm{T}=0 \\
u=u_{1}-u_{0}, \mathrm{~T}=0
\end{array}\right.
$$

The sliding problem caused by excavation unloading is transformed into the initial value problem of the ordinary differential equation, where the form of the governing equation of Equation (29) is similar to that of Equation (10), and the general solution of the equation is:

$$
u=C_{1} \cos (\sqrt{M} T)+C_{2} \sin (\sqrt{M} T)+\frac{N}{M}
$$

And then from the initial condition $u(0)=u_{1}-u_{0}$ Initial condition $\left.\frac{d u}{d T}\right|_{\mathrm{T}=0}=\frac{J}{\sqrt{I}} \sin \left(\sqrt{I} t_{1}\right)$, the Eq. (31) is obtained.

$$
\begin{aligned}
u= & \left(u_{1}-u_{0}-\frac{N}{M}\right) \cos (\sqrt{M} T) \\
& +\frac{J}{\sqrt{M I}} \sin \left(\sqrt{I} t_{1}\right) \sin (\sqrt{M} T)+\frac{N}{M}
\end{aligned}
$$

By substituting Equation $T=t-t_{1}$ into Eq. (31), the general solution of Eq. (29) can be obtained.

$$
\begin{aligned}
u= & \left(u_{1}-u_{0}-\frac{N}{M}\right) \cos \left(\sqrt{M} t-\sqrt{M} t_{1}\right) \\
& +\frac{J}{\sqrt{M I}} \sin \left(\sqrt{I} t_{1}\right) \sin \left(\sqrt{M} t-\sqrt{M} t_{1}\right)+\frac{N}{M}
\end{aligned}
$$

Where, $I, J, M$ and $\mathrm{N}$ can be solved by Eq. (8) and Eq. (26) respectively. The above equation is the displacement equation of the sliding body under the condition $u \in\left[u_{1}-u_{0}, u_{2}-u_{0}\right)$.

\section{2. 3 For $u \in\left[u_{2}-u_{0},+\infty\right)$}

Firstly, the duration and velocity of Eq. (32) at the point are calculated to serve as the initial conditions for the establishment of the displacement equation of this section $u=u_{2}-u_{0}$. Then, by substituting it into Eq. (32), it can be calculated that $u=u_{2}-u_{0}$.

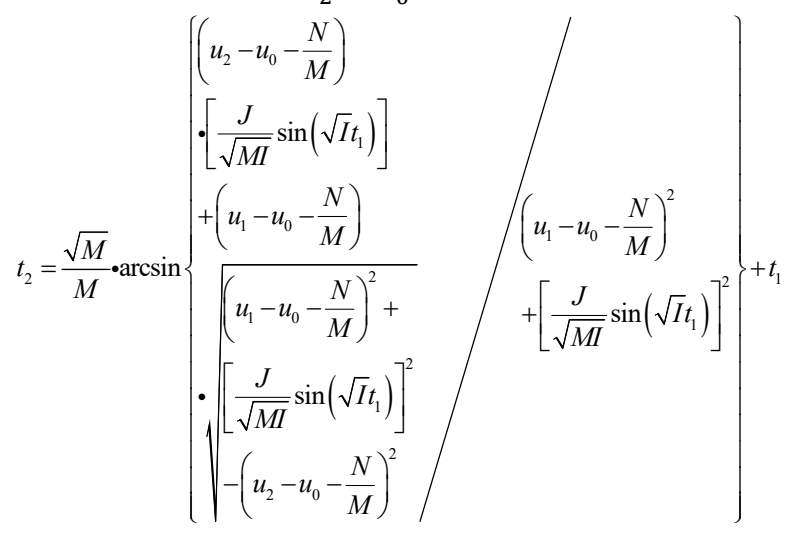

According to Eq. (33), the initial displacement velocity at this stage can be calculated as:

$$
\begin{aligned}
v\left(t_{2}\right)= & \frac{J}{\sqrt{I}} \sin \left(\sqrt{I} t_{1}\right) \sin \left(\sqrt{M} t_{2}-\sqrt{M} t_{1}\right) \\
& -\left(u_{1}-u_{0}-\frac{N}{M}\right) \sqrt{M} \sin \left(\sqrt{M} t_{2}-\sqrt{M} t_{1}\right)
\end{aligned}
$$

In the formula, $t_{2}$ is calculated according to Eq. (33), The sliding resistance that the structural plane can provide 
at this displacement stage is a constant value, so the displacement acceleration at this stage is a constant value, and the motion equation of the sliding body at this stage can directly give the displacement:

$$
\begin{aligned}
u= & {\left[\begin{array}{l}
\frac{J}{\sqrt{I}} \sin \left(\sqrt{I} t_{1}\right) \sin \left(\sqrt{M} t_{2}-\sqrt{M} t_{1}\right) \\
-\left(u_{1}-u_{0}-\frac{N}{M}\right) \sqrt{M} \sin \left(\sqrt{M} t_{2}-\sqrt{M} t_{1}\right)
\end{array}\right]\left(t-t_{2}\right) } \\
& +\frac{W \sin \beta-L k_{2} u_{2}+L k_{2} u_{1}-L k_{1} u_{1}}{2 m}\left(t-t_{2}\right)^{2}+u_{2}-u_{0}
\end{aligned}
$$

Summary Eq. (21), Eq. (32), Eq. (35) can get three lines model based on shear strengthening of excavation unloading conditions caused by the upper slope sliding displacement of rock mass structural plane control equation. The stability of the slope can be analyzed through results of convergence, and the state of slope stability and deformation stages can be determined through the results, which is calculated by the field displacement monitoring data and the theoretical comparison. Accordingly, a suitable time and way can be chosen for slope reinforcement. The displacement equation of the sliding body is:

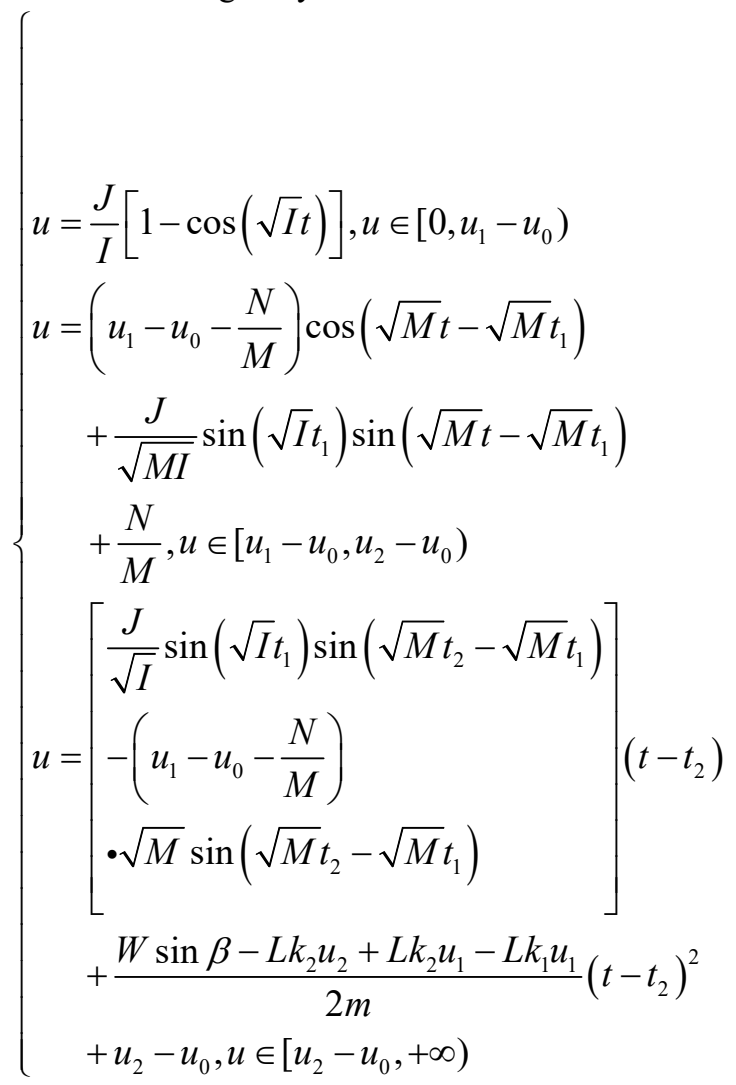

Where, $I, J, M$ and $N$ can be obtained by solving Eq. (8) and Eq. (26) respectively.

\section{Instance}

The sandstone in the middle of the cutting slope on the right side of an expressway in southwest China was cut by joint fissures. The collapse occurred along the sand-shale boundary the results calculated by the field displacement monitoring data and the theoretical comparison, with a collapse volume of about $1000 \mathrm{~m}^{3}$. The collapse body is a large block sandstone. After the instability, the sandstone block in the middle of the slope produces a blank surface with a thickness of about $8 \mathrm{~m}$, and the unloading crack appears along the joint fracture surface on the side slope surface, which is combined and cut together with the sandstone plane and joint fracture, resulting in the formation of isolation body of the sandstone in the middle slope.

\subsection{Calculation conditions}

According to the field survey results, the upper part of the slope top is sandstone, and the lower part is local mudstone interbedded sandstone strip, which belongs to the north east wing of Dongxi anticline. The occurrence of sandstone is $50^{\circ} \angle 23^{\circ}$, and the sandstone is tangential slope. However, the occurrence change of the contact surface between sandstone and mudstone is relatively significant, with the variation of $72 \sim 76^{\circ} \quad \angle 23 \sim 40^{\circ}$. The dip angle of the contact surface is steep in the front and slow in the back, forming the upper sandstone body to the sky. At the same time, according to the test results of wave velocity, the wave velocity also significantly decreases on the interface between sandstone and mudstone. The wave velocity decreases from the $2750 \sim 2880 \mathrm{~m} / \mathrm{s}$ to $1768 \sim 1961 \mathrm{~m} / \mathrm{s}$, indicating that there is a weak inter-layer on the interface between sandstone and mudstone, and this weak inter-layer is the integral controlling factor of the slope, and the whole slope is controlled by the structural plane. The original sand-mudstone contact surface is shown in figure 4.

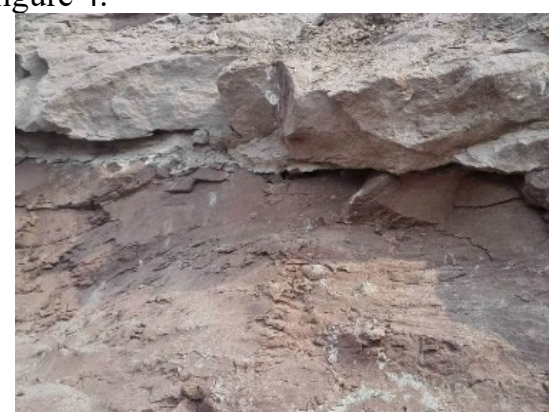

Fig.4. Contact surface of original sand and mudstone.

Collapsed cutting part are mainly sandstone, and the results calculated by the field displacement monitoring data and the theoretical comparison. Sandstone rock is composed of three groups of fractures and level combination of cutting and crack L1: $54^{\circ} \angle 61^{\circ}$, fissure L2: $235^{\circ} \angle 66^{\circ}$. and fissure L3: $144^{\circ} \angle 64^{\circ}$. Among them, the crack L1 and L 2 roughly along the slope tendency, fissure L3 along the slope to the development, sandstone blocks to tangential airport road direction is controlled by joint fissure L3 control gradually development unloading cracks, block along the sandstone and mudstone interface plane sliding failure. In this paper, the contact surface of sand and mudstone was selected as the main control structural plane for mathematical model verification, and the friction resistance generated by cracks L1 and L2 on the side of the slide body was ignored. This problem can 
be simplified into a two-dimensional calculation model, and a section at the position of the landslide axis in the middle of the slide body was selected as the calculation and analysis object, as shown in figure 5.

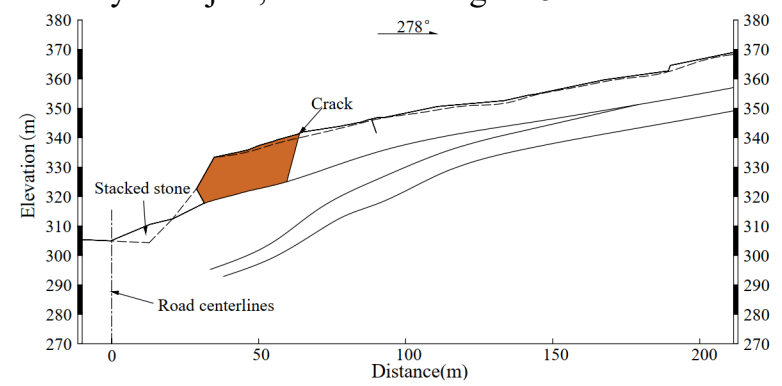

Fig. 5. Geological profile of the landslide.

In the process of slope instability and collapse, due to the instability and collapse of the leading block and the loss of support of the upper block, unloading cracks are produced in the back edge. The fractures have strong penetration and cut through the whole upper sandstone layer, and the fracture surface is flat and local mud is filled. Therefore, the anti-sliding force provided by the tensile strength of the trailing edge of the sliding body is not considered in the calculation of the sliding body. According to the field investigation, there is a softening layer on the contact surface between sandstone and mudstone, and some shearing mudstone layer can be seen on the contact surface after sliding, with a thickness of $2 \sim 3 \mathrm{~cm}$. The thickness of the interlayer is $2.5 \mathrm{~cm}$ here. According to the geological survey results, which calculated by the field displacement monitoring data and the theoretical comparison, the upper sandstone weight $\gamma$ is $24.2 \mathrm{kN} / \mathrm{m}^{3}$, the internal friction angle of sand and mudstone layer below the basement is $13.5^{\circ}$ and the cohesion is $31.0 \mathrm{kPa}$ in natural state $\varphi$. In addition, according to the geological section, the slope height is $29.0 \mathrm{~m}$, slope angle is $45.0^{\circ}$, overhead height is $13.5 \mathrm{~m}$, the slip surface length is $29.0 \mathrm{~m}$, and the slip surface inclination is $15.0^{\circ}$. The slide of leading edge sandstone is regarded as excavation unloading. When calculating the dangerous rock mass of the back sandstone, the unloading force of the frontage should be calculated. The potential sliding body (yellow block) caused by the instability slide of leading edge block (gray block in figure 5) is unloaded on the empty surface. In the calculation of unloading amount $F^{\prime}$, the leading edge instability slide block is treated as the retaining wall of the potential sliding body. According to Technical code for building slope engineering (GB 50330-2013) [16], the unloading load on the bare surface of the potential sliding body caused by the leading edge collapse can be calculated by the following formula, and the unloading load value is $241 \mathrm{kN}$.

$$
F^{\prime}=W \tan (\beta-\varphi)-\frac{c L \cos \varphi}{\cos (\beta-\varphi)}
$$

In the formula, $c$ is the cohesion of the weak structural plane $(\mathrm{kPa})$, and $\varphi$ is the internal friction Angle of the structural plane $\left(^{\circ}\right)$.

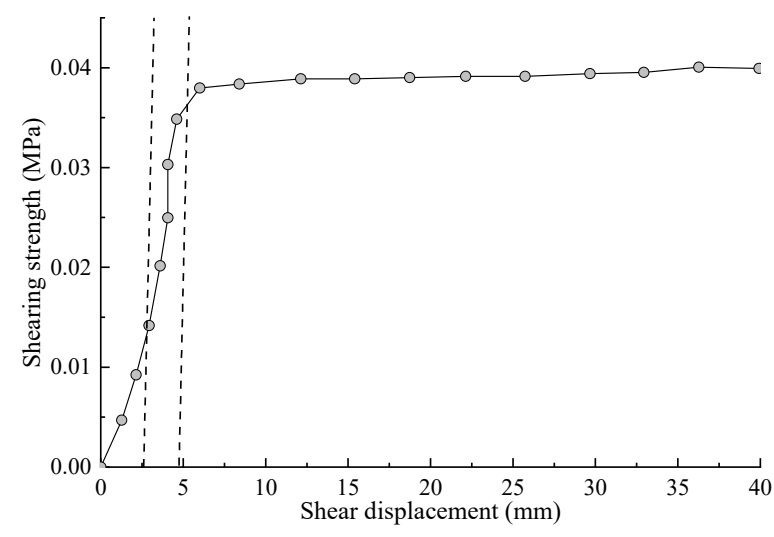

Fig. 6. Relationship curve between shear stress and shear displacement of structural plane.

According to the geological prospecting data and relevant test results (as shown in figure 6), the shear characteristics of structural plane materials conform to the three-fold line constitutive model of shear strengthening. The shear stiffness of structural plane at the initial shear stage is set at $6 \mathrm{MPa} / \mathrm{m}$, and that at the shear strengthening stage is set at $7.1 \mathrm{MPa} / \mathrm{m}$.

\subsection{Calculation and analysis}

In the stage of slope emergency rescue, relevant units adopt monitoring equipment to carry out wireless remote real-time monitoring of dangerous rock displacement. According to the location of the geological section selected by calculation, the response monitoring point is selected to verify the calculated results. The displacement results of dangerous rock mass monitored by the monitoring point are shown in the figure below.

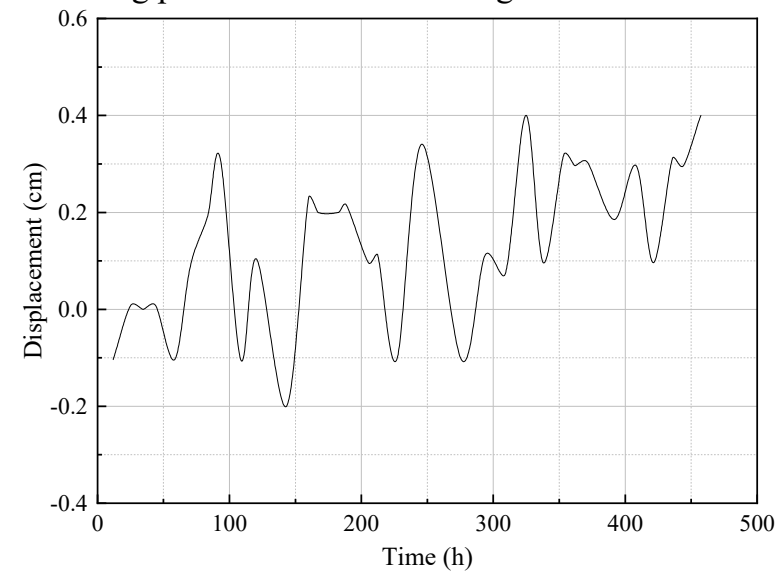

Fig. 7. Time-displacement increment curve of monitoring point (displacement monitoring of dangerous rock).

As the mathematical models of three line based on shears strengthening of unloading conditions caused by the upper slope sliding displacement of rock mass structural plane control Eq. (36) as the analytical solution of the model, the generation of each calculation parameter values into the day before, using Excel to landslide trailing edge sandstone of dangerous rock mass displacement process calculation. The calculated values and measured values for such are shown below. 


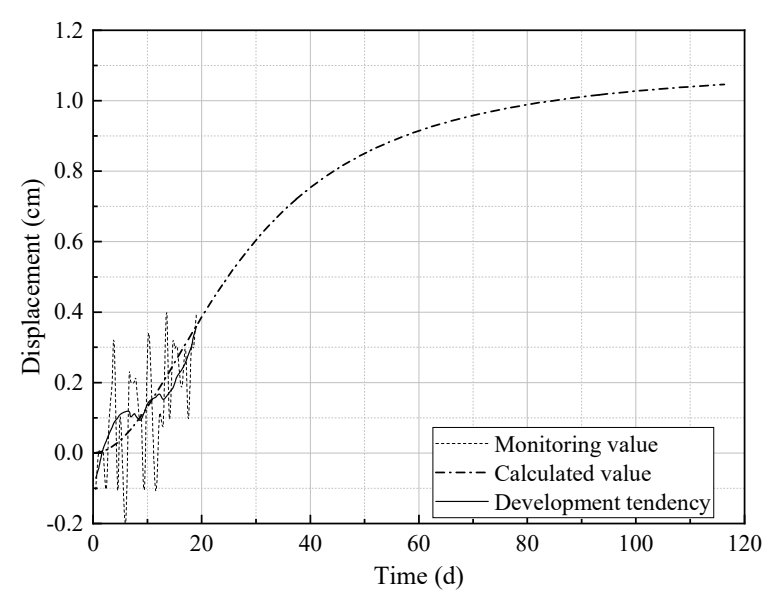

Fig. 8. Comparison of calculated and measured displacements of dangerous rocks at monitoring points.

Slump of front and cleaning up the upper sandstone as the process of excavation unloading, the slope of the structure plane position was established based on the above model for computing the selection of monitoring location development process from the front slump moment dangerous rock displacement calculation (according to the structural plane angle of actual displacement into horizontal displacement), and comparing with the results in the displacement monitoring, the results as shown in the above.

Field displacement monitoring data and results are affected by many factors, such as system error, environmental factors, etc, and the measured data fluctuate to a certain extent. Now, the second-order Savitzky-Golay convolution smoothing algorithm is adopted to smooth and denoise the monitored displacement data, and the development trend line of the measured displacement is shown as the solid line in figure 8 .

By comparing the calculated and measured values of the displacements of dangerous rock mass, it is found that the calculated displacement values can better simulate the displacement development process of dangerous rock mass, and can predict the displacement development trend to a certain extent. As shown in figure 8, after dangerous rock instability occurs, the upper opening of dangerous rock mass due to lose support at the front, along the main direction of the structural plane direction pointing at the slope foot together. Under the action of the slide and the upper exist a certain degree of decline in dangerous rock mass deformation, deformation monitoring results and calculation results can be used to reflect this shift. According to the development trend of the monitored displacement, the average horizontal displacement rate of the dangerous rock mass can be calculated to be approximately $0.0239 \mathrm{~cm} / \mathrm{d}$, while the average horizontal displacement rate during the monitoring period can be calculated to be $0.0193 \mathrm{~cm} / \mathrm{d}$ according to the calculated displacement, and the calculated value is close to the measured result. According to the displacement calculation curve, it can be seen that the calculated displacement value of the dangerous rock mass finally converges, and the dangerous rock mass will not fail. The dangerous rock mass will enter the displacement deceleration stage 48 days after the leading edge instability landslide, and the displacement will basically stop after $116 \mathrm{~d}$, and the final displacement of the dangerous rock mass is about $1.04 \mathrm{~cm}$.

\section{Summary}

In this paper, fully considering the influence of the deformation characteristics of structural plane and mechanical characteristics, establishing contains slide position shift and time under the dual function of excavation unloading of shear strengthening building three line constitutive model. Based on the moved slope deformation features of structure plane motion equation, the equations are solved, and obtain the analytical solution of displacement equation. Using the calculation method of a highway in southwest China induced displacement of slope excavation process is simulated and calculated results tally with the actual displacement monitoring data of basic, namely the calculation method can reproduce the instability of airport dangerous rock mass sliding process. The structural plane control based on the deformation characteristics provides a reference for slope stability.

\section{Acknowledgments}

Open Fund for Key Laboratory of Failure Mechanism and Prevention and Control Technology of Earth-rock Dam of Ministry of Water Resources (YK319006).

\section{References}

1. LIU, C.H. et al. Stability of layered rock slope. Science Press. (2012)

2. Wang, Z.Q., Jiang, Z.H. Failure mode and stability prediction of bedding rock slopes with steeply inclined structural surfaces under excavation conditions. The Chinese Journal of Geological Hazard and Control, 29(1): 40-45. (2018)

3. Chen, Q.F., Chen, D., Wei, C.S. Principle and discriminant method of structural plane connectivity. Journal of Geotechnical Engineering, 35(S2): 230-235. (2013)

4. Gu, D.Z. Foundation of rock Engineering Geo -mechanics. Science Press. (1979)

5. Wang, J.M., Chen, Z.H., Zhang, L.F., et al. Fracture mechanics analysis of instability of antidipping layered rock slopes. Chinese Journal of Computational Mechanics, 37(01): 75-82. (2020)

6. QI, S.W. Study on seismic permanent Displac e-ment of rock slope considering structural su rface degradation. Chinese Journal of Geotech nical Engineering, (03): 452-457. (2007)

7. Zhao, W, He, Y.L. Stability Evaluation of rock mass slope based on Case Reasoning. Journal of Railway Engineering, (7): 5-9. (2008) 
8. Xu, L., Ren, Q.W. Research on new constitutive model of rock structural plane. Rock and Soil Mechanics, 32(S1): 217-224. (2011)

9. Wan, Z.J. Study on the stability of multi-structural plane high and steep rock slope near dam reservoir bank. Journal of Three Gorges University (Natural Science edition), 41(S1): 112-115. (2019,) 